\title{
A lock-in-based method to examine the thermal signatures of magnetic nanoparticles in the liquid, solid and aggregated states $\uparrow$
}

\author{
C. A. Monnier, ${ }^{\text {a }}$ M. Lattuada, ${ }^{a}$ D. Burnand, ${ }^{\text {b }}$ F. Crippa, ${ }^{a}$ J. C. Martinez-Garcia, ${ }^{a}$ \\ A. M. Hirt, ${ }^{C}$ B. Rothen-Rutishauser, ${ }^{a}$ M. Bonmarin ${ }^{d}$ and A. Petri-Fink ${ }^{\star a, b}$
}

\begin{abstract}
We propose a new methodology based on lock-in thermography to study and quantify the heating power of magnetic nanoparticles. Superparamagnetic iron oxide nanoparticles exposed to a modulated alternating magnetic field were used as model materials to demonstrate the potency of the system. Both quantitative and qualitative information on their respective heating power was extracted at high thermal resolutions under increasingly complex conditions, including nanoparticles in the liquid, solid and aggregated states. Compared to conventional techniques, this approach offers a fast, sensitive and non-intrusive alternative to investigate multiple and dilute specimens simultaneously, which is essential for optimizing and accelerating screening procedures and comparative studies.
\end{abstract}

\section{Introduction}

Magnetic nanoparticles (NPs) are fascinating materials for designing nanoscale tools, as their behaviour can be tailored and remotely controlled. In this context, iron oxide NPs (SPIONs) are frequently used candidates owing to their superparamagnetic properties. ${ }^{1,2}$ These NPs generate heat when exposed to an alternating magnetic field (AMF) as a result of relaxation processes, ${ }^{1,3}$ which in turn render them intriguing for a wide range of applications. ${ }^{4-11}$ One notable implication of this feature is using the converted energy for medical purposes (e.g. for cancer therapy by means of magnetic fluid hyperthermia), ${ }^{8,12}$ and both research and development are currently ongoing to translate this technology to the bedside. ${ }^{7,8,13}$

Effective conversion of magnetic energy into heat is essential to keep administration dosages minimal and therapeutic efficiency maximal. This factor, usually represented in the literature by the Specific Adsorption Rate (SAR), ${ }^{2,14,15}$ is known to be dictated by many specific material properties, such as the NP type, size, polydispersity, surface or surface chemistry,

${ }^{a}$ Adolphe Merkle Institute, University of Fribourg, Chemin des Verdiers 4, 1700 Fribourg, Switzerland. E-mail: alke.fink@unifr.ch

${ }^{b}$ Chemistry Department, University of Fribourg, Chemin du Musée 9, 1700 Fribourg, Switzerland

${ }^{c}$ Institute of Geophysics, ETH Zurich, Sonneggstrasse 5, CH-8092 Zurich, Switzerland ${ }^{d}$ Institute of Computational Physics, Zurich University of Applied Sciences,

Technikumstrasse 9, 8400 Winterthur, Switzerland.

E-mail:mathias.bonmarin@zhaw.ch

$\dagger$ Electronic supplementary information (ESI) available. See DOI: 10.1039/ c6nr02066f crystallinity or state of dispersion..$^{3,14,16-21}$ Experimentally assessing the heating efficiency of the respective NPs is thus a prerequisite prior to their usage, given the numerous variables which may affect the energy output. Evidently, reliable and suitable analytical methods are required for this task.

With respect to magnetic NPs and their heating power, standard calorimetric methods, which rely on measuring the transient temperature rise of the sample as a function of time, are among the most straightforward and widely used approaches. ${ }^{2,22}$ The SAR can, amongst other methods, ${ }^{15}$ be deduced by assessing the initial heating slope (i.e., $\Delta$ temperature/ $\Delta$ time). ${ }^{15,22,23}$ Although increasingly popular, numerous uncertainties are linked to the experimental parameters (e.g. the AMF field strength and frequency) and data analysis methods (e.g. at what time point to set the fit), ${ }^{15}$ and are known to significantly affect the final outcomes. ${ }^{22-24}$ As a result, considerable divergence in SAR values can be observed in the literature, rendering direct comparison difficult between synthetic procedures, research groups and institutions. ${ }^{14,24}$

Differences are found between the used characterization sensors as well, with fiberoptic cables being the most represented in the field. ${ }^{2,22}$ Being "immune" to the effects of an AMF, they are ideal to record temperature variations over time within a (colloidal) solution. However, the data acquisition is one-dimensional, and single-point measurements cover only a small fraction of the sample, which renders the probe susceptible to selective positioning. ${ }^{22}$ As an example, it has been shown that reported SAR values, amongst others, depend on the positioning of the temperature sensor. ${ }^{22,25}$ Moreover, fiberoptic cables are invasive, implicating that any sample of 
interest needs to be compliant enough to be investigated. Thermal imaging - or thermography - is another option which provides a two-dimensional, ${ }^{26}$ non-invasive thermal overview of the sample surface, ${ }^{27}$ offering the possibility of investigating several samples at once along with controls. However, thermal waves from localized hotspots diffuse rapidly and blur the location of the heat sources. Moreover, heat losses to the surrounding occurring over time, either by convection or conduction, subsequently affect the sample temperature and limit the spatial resolution, ${ }^{28}$ accuracy, precision and clarity. In this respect, an analytical method which combines the aforementioned positive attributes with specificity, simplicity, objectiveness in data analysis and user-friendliness has not yet been reported, but would offer a considerably broader range of analytical and experimental opportunities.

A promising and rather unexplored alternative is offered by lock-in thermography (LIT), a methodology pioneered in 1984 which is based on the modulation of thermal radiation. ${ }^{29}$ In short, the heat-generating stimulus is periodically modulated over a specified number of cycles while an infrared camera continuously records the thermal events. The data acquisition and output are unlike other methods in that rather than recording variations of temperature over time, the amplitude, or the magnitude of the temperature oscillations during the modulation cycles, is determined. In turn, thermal signals unrelated to the induced heat (e.g. steady temperature drifts or reflections) are selectively filtered out. Temperature variations as low as $0.001 \mathrm{~K}$ can be detected, ${ }^{28,29}$ and lateral heat spreading is suppressed if the modulation frequency is set high enough. LIT with all its benefits is thus currently a standard method used to test composite materials and electronic components (e.g. solar panels). ${ }^{28,30}$ Though this method has not established itself in the field of magnetic NPs, it offers distinct advantages when addressing the previously mentioned concerns in investigating them. For example, magnetic NPs can be detected by LIT if the AMF is modulated. ${ }^{26,31}$ Whether quantitative data can be extracted from these measurements has, however, not been addressed to date.

In this study, we demonstrate that LIT can be used as both a qualitative as well as a quantitative tool to examine the thermal signatures of magnetic NPs. SPIONs exposed to a modulated AMF were chosen as model heat-generating NPs due to their versatility and prominence in medical research. This method is then applied to systematically screen their heating power by deducing the initial heating slope from the recorded amplitude images. By doing so, we show that quantitative data can be derived directly without any post-processing steps (e.g. fitting), thus avoiding the repetitively addressed uncertainties and subjectivity in data analysis. ${ }^{15,22}$ For validation, data are compared to results obtained by conventional methods (i.e., fiberoptic cables or standard thermography) and simulations, ${ }^{3}$ which highlight both the precision and sensitivity of the approach. The implication of these advantages is underlined by showing the thermal behaviour of these NPs of different sizes, concentrations and states of dispersion.
LIT yields a two-dimensional, contactless overview of the respective heating slopes in the regions of interest. This enables the investigation of multiple samples simultaneously, along with controls. We show as well that more complex samples, such as semi-solid or solid models, can be investigated while avoiding the previously addressed concerns. ${ }^{22}$ In return, the reliability of fiberoptic cables and two-dimensional features of thermal imaging devices are combined with LIT, which is further enhanced by rapidity and objectiveness. Given the need of using more standardized equipment and complex models, ${ }^{16,32}$ this method helps by predicting the thermal behaviour of NPs in any state of matter, which will consequently aid in enhancing their applicative value.

\section{Experimental methods}

\section{Iron oxide nanoparticles}

Iron oxide NPs coated with oleic acid were prepared by thermal decomposition. ${ }^{33,34}$ Prior to this, an iron oleate complex was prepared by boiling a mixture of iron chloride and sodium oleate in a $1: 3$ ratio overnight and subsequently washing it with Milli-Q water. Oleic acid and iron oleate were then mixed in a $250 \mathrm{~mL}$ round bottom flask and kept in a vacuum oven $\left(50^{\circ} \mathrm{C}, 2 \mathrm{~h}\right)$ prior to usage. The mixture was then thermally decomposed in high boiling solvents under inert atmospheres (argon) and magnetic stirring. Defined heating ramps were used for every synthesis (ESI $\dagger$ ). The respective NP sizes were tuned by using different reactant ratios (i.e., iron oleate: oleic acid ratios between 1.5:1 and 2.2:1), solvents (i.e., trioctylamine and docosan) and decomposition temperatures (i.e., 320 and $335^{\circ} \mathrm{C}$ ). The final temperature and the reaction time at the maximum temperature were varied according to the desired NP size. A detailed summary of all these parameters along with representative transmission electron micrographs is provided in the ESI. $\dagger$ The reaction was then cooled down quickly, resulting in a black-brownish suspension. The particles were then precipitated/washed three times with ethanol/hexane (5:1 volume ratio) and centrifuged (30 000g, 3 minutes) and finally re-suspended in hexane.

\section{Ligand exchange and surface polymer synthesis}

As a first step, oleic acid was exchanged with citric acid to render the hydrophobic NPs hydrophilic. ${ }^{35}$ The NPs were precipitated by ethanol and collected with a strong magnet, and then redispersed in a 1:1 DMF: DCB (volume ratio) solution. Citric acid was then added (1-2 $\mathrm{mg}$ per $\mathrm{mg}$ Fe) to the mixture, which was then heated at $100{ }^{\circ} \mathrm{C}$ for $24 \mathrm{~h}$. The particles were then precipitated again with ethanol and a strong magnet, washed three times with acetone and redispersed in Milli-Q water. 1-2 droplets of aqueous ammonia were added to render the solution optically homogeneous. As a second step, citric acid as a surface molecule was exchanged with catecholPEG-maleimide $(5 \mathrm{kDa}){ }^{36}$ The synthesis of the latter was adapted from a previously described method. ${ }^{37}$ A solution of dopamine hydrochloride (304 mg, $1.602 \mathrm{mmol}, 10.0$ eq.) and 
saturated $\mathrm{NaHCO}_{3}$ was degassed with argon for 10 minutes. Maleimide-PEG-carbonate-NHS (630 mg, $0.1602 \mathrm{mmol}, 1$ eq.) was then added portionwise. The reaction was stirred for three hours at room temperature under an inert atmosphere (argon). The polymer was then purified by dialysis overnight. The following day, the dialysis content was acidified to $\mathrm{pH}$ 1-2 with $1 \mathrm{M} \mathrm{HCl}$, and then extracted three times with $\mathrm{CHCl}_{3}$. The combined organic layers were dried over $\mathrm{MgSO}_{4}$ and precipitated three times in ice-cold hexane (final yield $=244 \mathrm{mg}$, $30 \%$ ). For subsequent ligand exchange, the polymer was dissolved in Milli-Q water. Iron oxide NPs coated with citric acid were then mixed with a catechol-PEG-maleimide solution (10 mg of Cat-PEG-Mal per mg Fe). The mixture was then sonicated overnight at room temperature. Excess polymer was removed by dialysis.

\section{Nanoparticle characterization}

Iron concentrations were determined with an Optima 7000 DV inductively coupled plasma optical emission spectrometer (ICP-OES, PerkinElmer). The NP suspensions were dissolved in $37 \% \mathrm{HCl}$ solutions overnight and investigated the following day. The core diameters were determined by transmission electron microscopy (TEM). The NP suspensions were dried on copper carbon-coated mesh grids (300 mesh, Plano GmbH) and investigated with a FEI Tecnai F20 transmission electron microscope operating at $200 \mathrm{kV}$. The acquired micrographs were then used to determine the diameter of the various batches by automated size distribution analysis (ImageJ, v. 1.46r).

Hysteresis and First-Order-Reversal Curve (FORC) measurements were performed using a Princeton Measurements Corporation (PCM) vibrating sample magnetometer (VSM), model 3900. $30 \mu \mathrm{L}$ of sample solution $(5.0 \mathrm{mg}$ Fe per $\mathrm{mL}$ ) were used. Low temperature measurements were made on the same instrument with the PCM cryostat. Hysteresis loops were made using a measurement averaging time of $100 \mathrm{~ms}$, and a variable field increment. The field increment was 5 Oe (307.89 $\mathrm{A} \mathrm{m}^{-1}$ ) in $H<20$ Oe or $1.592 \mathrm{kA} \mathrm{m}^{-1} ; 20$ Oe $(1591.55$ $\left.\mathrm{A} \mathrm{m}^{-1}\right)$ steps in intermediate fields $(200-2000 \mathrm{Oe})$, and 100 Oe (7.957 $\mathrm{kA} \mathrm{m}^{-1}$ ) above 2000 Oe (159.155 $\left.\mathrm{kA} \mathrm{m}^{-1}\right)$. FORC diagrams were made using 140 first-order reversal curves (increment of $30 \mathrm{Oe}, 2.39 \mathrm{kA} \mathrm{m}^{-1}$ ). Samples were first saturated in a $10 \mathrm{kOe}$ field $\left(795.7 \mathrm{kA} \mathrm{m}^{-1}\right)$, and then ramped back to the reversal field to measure the reversal curve. Data were processed using the MatLab code described by Winklhofer and Zimanyi. ${ }^{38}$

\section{Thermal measurements, SAR and ILP calculations}

The alternating magnetic field was generated by using a commercial coil system (Magnetherm ${ }^{\mathrm{TM}} \mathrm{V} 1.5$, nanoTherics Ltd) comprising a water-cooled coil, a function generator (SFG-2004, GW Instek) and a laboratory power supply (EA-PS 3032-20B, EA Elektro Automatic). The device was set to operate at $110 \mathrm{kHz} / 18.0 \mathrm{mT}$ and $535 \mathrm{kHz} / 12.5 \mathrm{mT}$ respectively. The infrared camera (Onca-MWIR-InSb-320, XenICs) was mounted on a standard microscope stand (Leica Microsystems) to allow a precise positioning and reduce mechanical vibrations coming from the Stirling motor-based camera cooling system (ESI Fig. $2 \dagger)$. The InSb array $(320 \times 256$ pixels $)$ recorded infrared radiation between 3 and $5 \mu \mathrm{m}$ at an image resolution of $0.017 \mathrm{~K}$ and a full frame rate of up to $250 \mathrm{~Hz}$. A Digital-to-Analogue acquisition card (DAQ) was used to generate the amplitude modulation signal. The acquired infrared images were transferred in real-time to a personal computer via a CameraLink-based frame grabber. All measurements were performed at modulation frequencies of $0.5 \mathrm{~Hz}$.

For all standard measurements, either the infrared camera was used or a Luxtron FOT Lab Kit fiberoptic cable system $(f=$ $1 \mathrm{~Hz}$, three individual probes) were used to record temperature changes over time exposed to the AMF. The fiberoptic cables were calibrated in $20^{\circ} \mathrm{C}$ Milli-Q water prior to every measurement. Changes in temperature were measured over a timescale between 120 and $180 \mathrm{~s}$. A linear regression fit was applied to the initial five measurement seconds to assess the heating slope $\beta$.

The heating slope $\beta$ was then used to calculate the SAR using the following equation:

$$
\mathrm{SAR}=\beta \times \frac{c_{\mathrm{P}}}{m_{\mathrm{Fe}}}
$$

$c_{\mathrm{p}}$ being the heat capacity of the solution and $m_{\mathrm{Fe}}$ the mass of magnetic material within it. ${ }^{14,15,22}$ These values were then normalized to the AMF frequency and square of the magnetic field strength to obtain the ILP. ${ }^{13}$

\section{Nanoparticles in biological media}

SPIONs $(d=21.7 \pm 0.9 \mathrm{~nm})$ coated with either citric acid or catechol-PEG-maleimide were diluted down to a concentration of $0.5 \mathrm{mg}$ Fe per $\mathrm{mL}$ in Milli-Q with/without Fetal Bovine Serum (FBS, 10\%), phosphate buffered saline (PBS) with/ without FBS and Dulbecco's Modified Eagle Medium (DMEM) with/without FBS (10\%). The suspensions were kept at $37{ }^{\circ} \mathrm{C}$ for $24 \mathrm{~h}$. Following this, the suspensions were investigated by LIT at a field frequency/strength of $535 \mathrm{kHz} / 12.5 \mathrm{mT}$. They were subsequently examined by TEM to assess their condition.

\section{Nanoparticles embedded in polyacrylamide gel}

SPIONs ( $d=21.7 \pm 0.9 \mathrm{~nm}$, coated with citric acid), 30\% acrylamide/bis solution and tetramethylethylenediamine (TEMED) and Milli-Q water (see ESI for ratios $\dagger$ ) were mixed and stirred in a round bottom flask for 10 minutes. Following this, the solution was split into two Eppendorf tubes (500 $\mu \mathrm{L}$ each), to which fresh ammonium persulfate (APS, 10\%, $5 \mu \mathrm{L}$ ) was added. $100 \mu \mathrm{L}$ of the polymerization mixture was then sandwiched between two cover slips (one has been functionalized with 3-(trimethoxysilyl)-propylmethacrylate, the other one is not). Gelation began after five minutes and was complete after one hour. Finally, the non-functionalized cover slip was removed, and the gel was washed in Milli-Q water for three days under gentle agitation. All gels were subsequently investigated at a magnetic field frequency/strength of $535 \mathrm{kHz} /$ $12.5 \mathrm{mT}$. 


\section{Mathematical model}

Among the several approaches proposed in the literature to compute the energy dissipated by magnetic NPs when exposed to an AMF, we chose the Linear Response Theory (LRT) formulated by Rosensweig. ${ }^{3}$

According to the LRT, it is assumed that the magnetization $M$ is proportional to the applied field $H$ :

$$
M=\chi H=\left[\left(\chi^{\prime}-\mathrm{i} \chi^{\prime \prime}\right) \mathrm{e}^{\mathrm{i} \omega t} H_{0}\right]
$$

In eqn (1), the applied field is a periodic function of time, and the magnetic susceptibility $\chi$ is formulated as a complex function in order to account for an in-phase and an out-ofphase component with respect to the magnetic field. The power per unit volume dissipated is given by the product of the energy dissipated during one cycle (area contained in the hysteresis loop) multiplied by the frequency. After some calculations, the final expression is:

$$
P=-f \mu_{0} \oint M \mathrm{~d} H=\pi \mu_{0} H_{0}{ }^{2} \chi^{\prime \prime} f
$$

The following relation for the dynamic susceptibility is used in the LRT:

$$
\chi=\frac{\chi_{0}}{1+2 \mathrm{i} \pi f \tau} \rightarrow\left\{\begin{array}{l}
\chi^{\prime}=\chi_{0} \frac{1}{1+(2 \mathrm{i} \pi f \tau)^{2}} \\
\chi^{\prime \prime}=\chi_{0} \frac{2 \pi f \tau}{1+(2 \mathrm{i} \pi f \tau)^{2}}
\end{array}\right.
$$

The equilibrium susceptibility is obtained from Langevin's model:

$$
\chi_{0}=\chi_{\mathrm{i}} \frac{3}{\xi}\left(\operatorname{coth}(\xi)-\frac{1}{\xi}\right)=\frac{\mu_{0} M_{\mathrm{s}}^{2} V_{\mathrm{p}}}{3 k T \rho_{\mathrm{p}}} \frac{3}{\xi}\left(\operatorname{coth}(\xi)-\frac{1}{\xi}\right)
$$

In eqn (4), $M_{\mathrm{s}}$ is the saturation magnetization of the particle, $V_{\mathrm{p}}$ the core particle volume, and $\rho_{\mathrm{p}}$ the particle density and $\xi$ is defined as:

$$
\xi=\frac{\mu_{0} M_{\mathrm{s}} V_{\mathrm{p}} H_{0}}{k T}
$$

The decay time $\tau$ is a combination of two characteristic times, one from Brownian relaxation $\left(\tau_{\mathrm{B}}\right)$ and the other for Néel relaxation $\left(\tau_{\mathrm{N}}\right)$

$$
\begin{gathered}
\frac{1}{\tau}=\frac{1}{\tau_{\mathrm{B}}}+\frac{1}{\tau_{\mathrm{N}}} \rightarrow\left\{\begin{array}{l}
\tau_{\mathrm{B}}=\frac{3 \eta V_{\mathrm{H}}}{k T} \\
\tau_{\mathrm{N}}=\frac{\sqrt{\pi}}{2} \tau_{0} \frac{\exp (\sigma)}{\sqrt{\sigma}}=\frac{\sqrt{\pi}}{2} \tau_{\mathrm{D}} \frac{\exp (\sigma)}{\sigma \sqrt{\sigma}} \\
\sigma=\frac{K_{\mathrm{V}} V_{\mathrm{p}}}{k T}, \quad \tau_{\mathrm{D}}=\tau_{0} \sigma
\end{array}\right.
\end{gathered}
$$

In eqn (6), $\sigma$ is the dynamic viscosity of the liquid surrounding the particle, $V_{\mathrm{H}}$ the hydrodynamic volume of the particle, and $K_{\mathrm{V}}$ the anisotropy constant of magnetocrystalline origin (identifying the direction of the easy axis of magnetization). The characteristic time constant $\tau_{0}$ is assumed to be equal to $\sim 10^{-9} \mathrm{~s}$.
Since the particles used in this work have a finite polydispersity, eqn (2) needs to be averaged over all particles, in order to obtain the total power generated $P_{\mathrm{T}}$ per unit particle mass:

$$
P_{\mathrm{T}}=\pi \mu_{0} H_{0}^{2} f \int \chi^{\prime \prime}(R) g(r) \mathrm{d} R
$$

where $g(R) \mathrm{d} R$ is the volume fraction occupied by particles with a radius between $R$ and $R+\mathrm{d} R$.

In order to simulate the time change of the temperature profile during a magnetic heating experiment, the following differential equation is used:

$$
c \frac{\Delta T}{\Delta t}=P_{\mathrm{T}} w
$$

In eqn (8), $c$ is the heat capacity of the particle suspension, and $w$ the mass fraction of particles in the suspension. The heat capacity of the particle suspension is computed as a weighted average of water and particles heat capacities.

Eqn (8) is used when heat transfer of the suspension with the surrounding environment is negligible. Even though $P_{\mathrm{T}}$ depends on temperature, it turns out that the temperature profiles computed by means of eqn (8) are almost linear in time. Since some experimental data, especially those involving large particles and higher concentrations, show a profiles that are strongly nonlinear, a heat transfer mechanism is introduced. Then, eqn (8) is modified as follows:

$$
c \frac{\Delta T}{\Delta t}=P_{\mathrm{T}} \mathcal{W}+h\left(T_{0}-T\right)
$$

where $h$ is the heat transfer coefficient and $T_{0}$ the outer temperature, which is assumed to be equal to the room temperature. The heat transfer coefficient is treated as a fitting parameter, since the exact mechanism for heat transfer is not well known.

Most of the parameters required in the simulations have been obtained experimentally. The results of the simulations indicate that the LRT can adequately predict the experimentally measured temperature profiles, as well as the SAR values. However, two parameters cannot easily be accessed experimentally and have been adapted in order to achieve such a good behaviour: the heat transfer coefficient has been varied, since the exact nature of the heat transfer mechanism is difficult to predict a priori, and the magnetocrystalline anisotropy constant $K_{\mathrm{V}} . K_{\mathrm{V}}$ has been found to be not only a function of the particle size, which is not surprising given that different particle sizes also have different saturation magnetization values, but also a function of the frequency, especially for the particles that produce the largest amount of heat. For both the smallest and the largest particles, instead, the amount of heat produced is so low that no reliable value can be estimated. While similar effects have already been observed in the literature, the physical explanation is not entirely clear, and might underline an intrinsic limitation of the LRT. All the applied values are summarized in the ESI. $\dagger$ 


\section{Results}

Standard calorimetric methods, independent of the sensor type, generally rely on recording the temperature changes of the sample over time (Fig. 1A). Measurements can be performed under both adiabatic or non-adiabatic conditions, the former being usually more time-consuming ${ }^{22}$ and the latter less accurate. ${ }^{23}$ These data are then typically used to calculate the slope of the heating curve (i.e., $\Delta$ temperature/ $\Delta$ time, $\beta$ ), which is assessed within the first few seconds of the measurement when heat losses to the surroundings are negligible (Fig. 1A). ${ }^{15}$ When dealing with magnetic NPs, this parameter is often used to calculate the SAR, and thus gives an account of their biomedical effectiveness. ${ }^{15}$ However, it is known that this approach may be misleading, ${ }^{15}$ as the final results may vary considerably between the selected fits and time points (ESI Fig. $1 \dagger) .{ }^{14,25}$ This is especially prominent in samples with weak signals, leaving a degree of subjectivity in the data analysis. Additional uncertainties can add up when considering the probes themselves: fiberoptic cables, for example, are invasive and depend on where they are placed, ${ }^{22}$ and infrared cameras are very susceptible to disruptive signals arising from lateral heat dissipation, reflections and thermal drift (Fig. 1B).

With lock-in thermography, the functional principle is fundamentally different. ${ }^{29}$ This method assumes that the external stimulus (i.e., the AMF) is periodically modulated at a constant frequency (Fig. 1C), while infrared images are synchronously recorded and stacked. The stack is then Fourier-transformed, resulting in a two-dimensional amplitude image which is evaluated at the modulation frequency (Fig. 1D). This amplitude

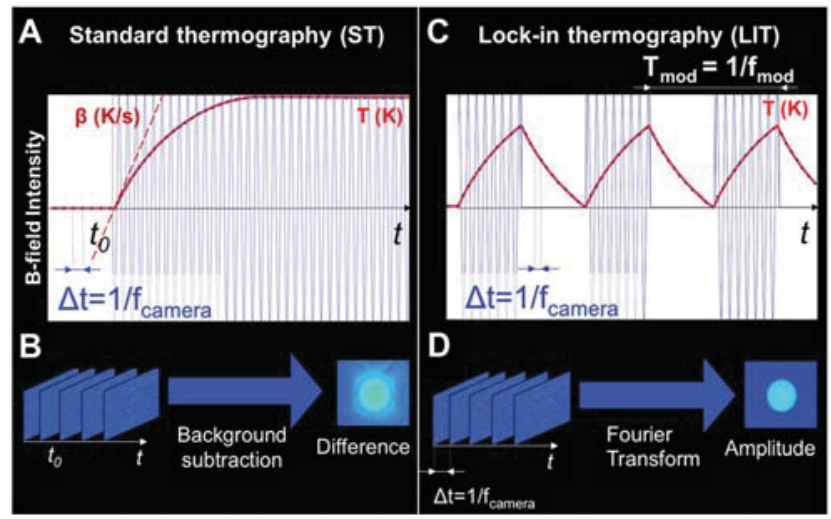

Fig. 1 A general comparison between the standard and lock-in thermography concepts. In standard thermography (ST), the transient temperature rise of the sample is tracked over time (A). The probe, for example an infrared camera, continuously records the overall change in temperature (B). In accordance with these data, the initial heating slope $\beta$ is fitted and extracted (ESI Fig. $1 \dagger$ ), which in turn reflects the heating power of the NPs. In lock-in mode (LIT), the stimulus, and thus the thermal emission signal, is periodically modulated (C). The signal is then Fourier-transformed to extract an image rendering the amplitude of the thermal modulations (D). From these averaged data, the initial heating slope can be computed at a very high degree of accuracy without the need for any fitting procedures. signal describes the magnitude of the sample surface temperature oscillations that would result from a perfectly harmonic AMF modulation, and is proportional to the dissipated power at the sample surface. ${ }^{28}$

Fourier transformation is computationally demanding. Therefore approximation methods are frequently favoured, as the amplitude needs to be evaluated at only one frequency (i.e., the modulation frequency). Different algorithms are employed and described in the literature. ${ }^{39}$ In our setup, the temperature measurement is synchronously recorded with the magnetic field amplitude modulation, and the frequency is set at a low level compared to the infrared camera frame rate (i.e., $0.5-2 \mathrm{~Hz}$ versus $100-250 \mathrm{~Hz}$ ). Under these conditions, synchronous narrow two-channel correlation is the optimal digital lock-in algorithm. ${ }^{28}$ The advantage of this method is that the transient temperature images do not need to be stored on the computer or camera memory, and the correlation can be achieved in real time. This way, the measurement can be stopped as soon as the desired sensitivity is reached by tuning the measurement duration (ESI $\dagger$ ).

In summary, the lock-in demodulation formalism works under the assumption that the measurement is performed under quasi-steady-state conditions, i.e., the sample surface temperature oscillates with time around a steady mean temperature. In practice, at the beginning of the modulation, the temperature varies during an initial non-steady-state phase. This initial period, or thermal relaxation time, will induce an amplitude shift in the demodulated signal. ${ }^{40}$ Theoretically, the quasi-steady-state should be reached before demodulating the IR images; however, this takes several minutes and consequently slows down the measurement process in practice. To solve this problem, we chose the solution introduced by Gupta and Breitenstein, ${ }^{40}$ in which errors caused by temperature drift are compensated by a simple correction formula based on the measurement of the temperature drift image. This method has the advantage that it can be achieved on-line without the need of storing all the infrared images in the computer memory.

To highlight the utility of this method for these particular applications, iron oxide NPs were chosen as model stimuliresponsive nanomaterials due to their ability to convert magnetic energy from an AMF into heat. Very narrow size distributions and high circularity were required to ensure an accurate correlation between diameter and heating efficiency. In this regard, four NP batches with different core sizes were prepared by thermal decomposition (Fig. 2A). ${ }^{33,34}$ The respective core sizes were tuned by varying the synthetic conditions, amongst others the precursor ratios and boiling solvents (ESI $\dagger$ ). Phase transfer to water was achieved via a two-step ligand exchange in order to obtain monodisperse NPs instead of clusters. ${ }^{41}$ Exchanging oleic acid with citric acid (Fig. 2B) ${ }^{35}$ was the first step, which was followed by a custom-made catechol-PEG-maleimide polymer (Fig. 2C) to ensure a tight grafting of the surface molecule to the NP surface. ${ }^{36}$ These NPs were used for all subsequent thermal measurements without any further modification. Prior to any thermal measurement, the magnetic and structural properties of all NP populations 


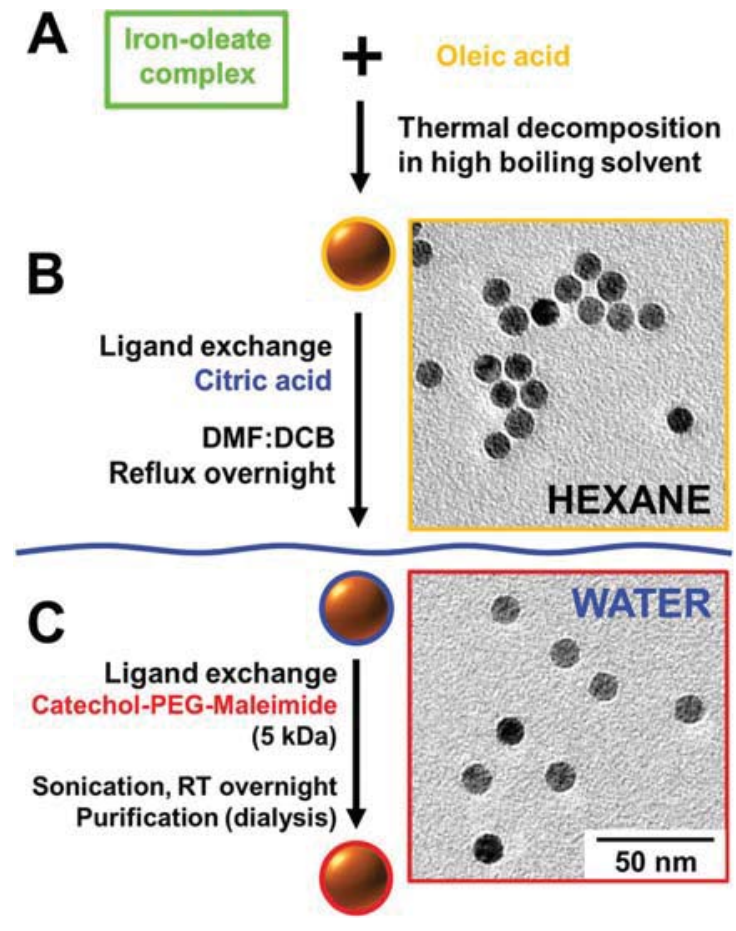

Fig. 2 SPION synthesis and transfer to water. Hydrophobic iron oxide NPs coated with oleic acid were prepared by thermal decomposition (A) and transferred to water by a two-step ligand exchange (B, C). First, the surface ligand was replaced with citric acid, after which the NPs were re-dispersed in water (B). In a second step, citric acid was replaced with PEG-catechol-maleimide (C) to provide a more stable and reliable surface coating molecule for all subsequent experiments.

were investigated in detail (Fig. 3A-C, ESI Fig. 3 and $4 \dagger$ ). The core diameters (Fig. 3A) were determined to be $12.8 \pm 0.6 \mathrm{~nm}$, $17.2 \pm 0.7 \mathrm{~nm}, 21.7 \pm 0.9 \mathrm{~nm}$ and $50.3 \pm 3.6 \mathrm{~nm}$ (Fig. 3B, average $\pm \mathrm{SD}, n=200$ ), giving a broad investigative range with respect to the size dependence of the heating power. Subsequent investigations by dynamic light scattering (DLS) confirmed that the NPs were monodisperse, with an overall polymer shell between 6 and $11 \mathrm{~nm}$ in thickness (ESI Fig. $4 \dagger$ ). Hysteresis loops were further recorded at $250 \mathrm{~K}$ (Fig. 3C). NPs below $22 \mathrm{~nm}$ in diameter were superparamagnetic, which was confirmed by FORC analysis (ESI Fig. 4B $\dagger$ ). The largest NPs $(d=50.3 \pm 3.6 \mathrm{~nm})$ had a significant coercivity, i.e., were magnetically ordered.

In a preliminary test, concentrated suspensions (i.e., $5.0 \mathrm{mg}$ Fe per $\mathrm{mL}$ dispersed in Milli-Q water) of all four NP diameters were loaded into specific cassettes (height $=6 \mathrm{~mm}, r=3 \mathrm{~mm}$, solution depth $=4 \mathrm{~mm}$, Fig. $3 \mathrm{D}$ ). Milli-Q water was added to every array as a negative control. All samples were simultaneously studied using two different field strength/frequency settings (i.e., $110 \mathrm{kHz} / 18.0 \mathrm{mT}$ and $535 \mathrm{kHz} / 12.5 \mathrm{mT}$ ). Conventional thermal images recorded by standard thermography (Fig. 3E, top) highlighted the limitations of this approach, as a clear pattern regarding the various NP diameters was hardly distinguishable after only several seconds (i.e., $10 \mathrm{~s}$ ) of measurement. The strong infrared reflections within the recorded space were too substantial, and progressively worsened over the duration of the measurement. In lock-in mode however, the individual samples became immediately apparent (Fig. 3E, bottom), and even the smallest thermal variations were visible. At the same time, all five controls remained invisible, showing that the observed thermal variations originated from the NPs alone. The amplitude values of every batch were then extracted and plotted (Fig. 3F), further highlighting the dependence of the NP core size ${ }^{18}$ on the effective heat conversion. While small NPs $(d=12.8 \pm 0.6 \mathrm{~nm})$ remained only sparsely visible, which agrees with their size-dependent heating capabilities, ${ }^{18}$ the thermal signal originating from the larger ones $(d=21.7 \pm 0.9 \mathrm{~nm})$ was significantly higher (Fig. 3D), but decreased again with the ultra-large diameter $(50.3 \pm 3.6 \mathrm{~nm})$. These observations are in agreement with the Linear Response Theory (LRT), as the overall diameter dictates the relaxation times, either Néel or Brownian, involved in heat dissipation. ${ }^{3}$ The acquired results were reproducible and consistent over a broader dilution range (ESI Fig. $5 \dagger$ ).

The quantitative value of these data was explored as well and compared to conventional methods. IR- and fiberoptic cable-based thermal measurements were made along with LIT for three different NP sizes and three increasingly dilute iron concentrations (i.e., 5.0, 2.5 and $1.0 \mathrm{mg}$ Fe per $\mathrm{mL}$, ESI Fig. $6 \dagger)$. With regard to the standard approaches, the initial heating slopes were evaluated by performing a least-squares linear fit to the acquired data ${ }^{23}$ (i.e., the first five measurement seconds), whereas those from LIT were directly deduced from the demodulated amplitude signals.

A consistent relationship between the experimental techniques was observed when comparing the different methods, independent of whether they are invasive or not (i.e., between fiberoptic cable- and standard thermography-based measurements). Although the heating slope values extracted by LIT were very similar to those obtained by both standard techniques (Fig. 4A and B and ESI Table $1 \dagger$ ), they showed predominantly the best agreement with the simulated values when averaged over all measurements. When normalized to iron concentrations, this agreement became even more apparent (Fig. 4C) in that standard deviations were significantly lower and sensitivity was higher (i.e., a direct consequence of the averaging nature and high sensitivity of the technique). Especially for low concentrations, LIT outperformed its counterparts. In turn, the recorded heating slopes (ESI Table $1 \dagger$ ) were used to determine the SAR (Table 1) and ILP ${ }^{13}$ (ESI Table $2 \dagger$ ). Interestingly, the results of the simulations indicated that, under the probed conditions and for these respective NPs, Néel relaxation was the mechanism responsible for heat dissipation over all particle sizes. In addition, the fitted magnetocrystalline anisotropy constant values decreased as the particle size increased, a trend that has previously been reported in the literature, ${ }^{42}$ even though the values used here were considerably lower than that of bulk magnetite.

With regard to heating effectiveness, although NP diameter is an important factor, the operating conditions are another. When employed in biological applications, NPs will unavoid- 

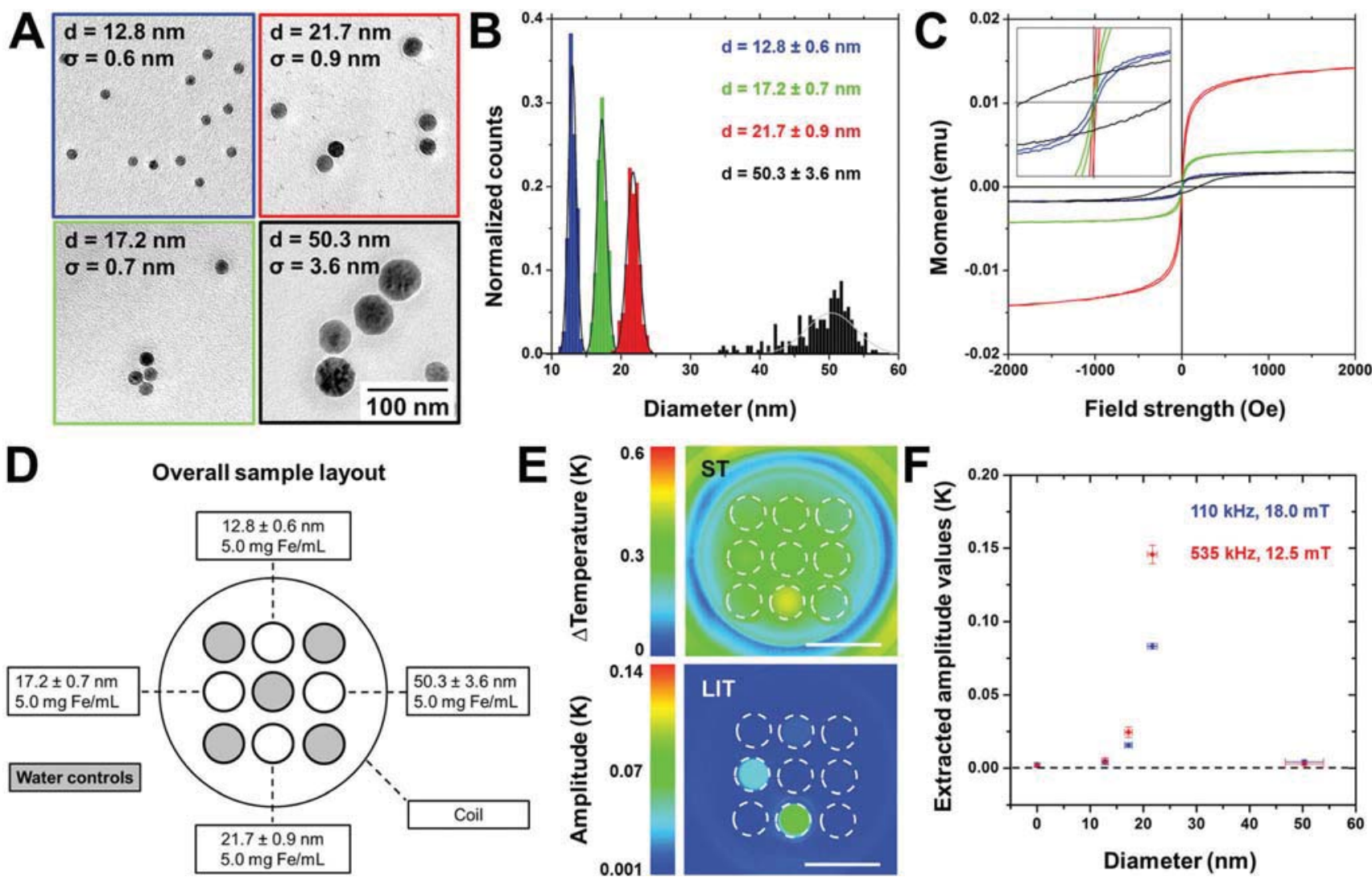

Fig. 3 Investigating SPIONs of distinct sizes and their thermal signatures by lock-in thermography. Four NP suspensions of increasing diameters were prepared, functionalized and investigated by TEM (A). Their core diameters and particle size distribution were then deduced from these micrographs (B). Hysteresis measurements were performed as well to show the magnetic properties of these respective NPs (C). These same NPs were all simultaneously investigated by LIT by loading them into individual cassettes along with water controls (D). Standard thermal imaging recordings were made on these arrays after being placed within the magnetic coil. However, the individual wells were not distinguishable anymore after a few seconds of measurement, as the blurring effects became too significant (E, top). In lock-in mode, all signals unrelated to NP heat generation were suppressed (E, bottom), yielding the distinct heat signatures originating from the NPs in their respective wells (E). These corresponding amplitude values were then extracted from LIT captions recorded at two different field strengths and frequencies and plotted as a function of the NP diameter (F).

ably interact with biomacromolecules (e.g. proteins, lipids etc. $)^{43}$ and electrolytes, which all exert a broad range of forces on them and define their physical properties. ${ }^{44}$ In this regard, the guiding factor is colloidal stability: when compromised the NPs aggregate, ${ }^{45}$ which impacts not only the physicochemical and magnetic properties, but also the experimental results and reproducibility. ${ }^{46}$ When testing NPs in water, these factors are not reflected. Investigating them in physiological surroundings is thus critical to correctly interpret data and validate the materials and their surface chemistry for subsequent application. The LIT method was thus applied for this task and used to monitor changes in the NP heating power and screen an array of differently coated NPs. These NPs were coated with either citric acid (Fig. 5A), a surface molecule which leads to a moderate degree of aggregation (i.e., a threefold increase of diameter from the non-aggregated state) in biological or physiological environments, ${ }^{47}$ or catechol-PEG-maleimide (Fig. 5B), with which the NPs show no sign of aggregation over prolonged time periods. ${ }^{44}$ Both models were diluted in commonly used and increasingly complex biological media, whereby the TEM and LIT measurements were performed after $24 \mathrm{~h}$ of incubation. Subsequent TEM imaging confirmed the presence of aggregates in all compromised samples (Fig. 5A, center and right, in comparison to the ones diluted in Milli-Q water), and was further confirmed by visible sedimentation in the suspensions. The NPs with catecholic anchors however were still perceived as monodisperse by TEM (Fig. 5B) and remained optically isotropic in their respective media for weeks. Interestingly, these observations could be directly linked to the LIT investigations: NPs which aggregated in biological media (i.e., with a citric acid coating) entirely lost their thermal signal (Fig. 5C and D). On the other hand, SPIONs coated with catechol-PEG-maleimide, which remained stable and monodisperse, ${ }^{36}$ did not lose their heating power over the entire buffer scale. In return, the quality of the respective NP coating molecule and its resilience under more challenging conditions were effectively shown in a standardized way without any additional instrumentation. 
A

$110 \mathrm{kHz}, 18.0 \mathrm{mT}$
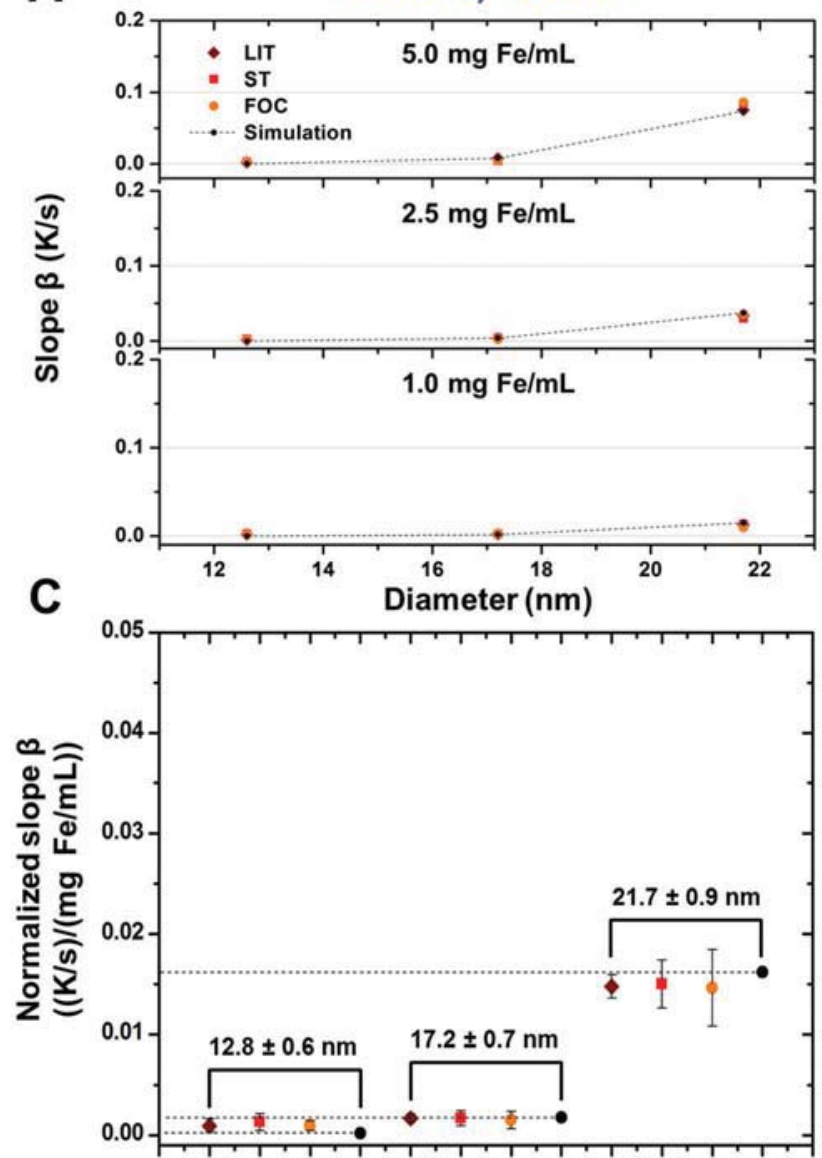

B
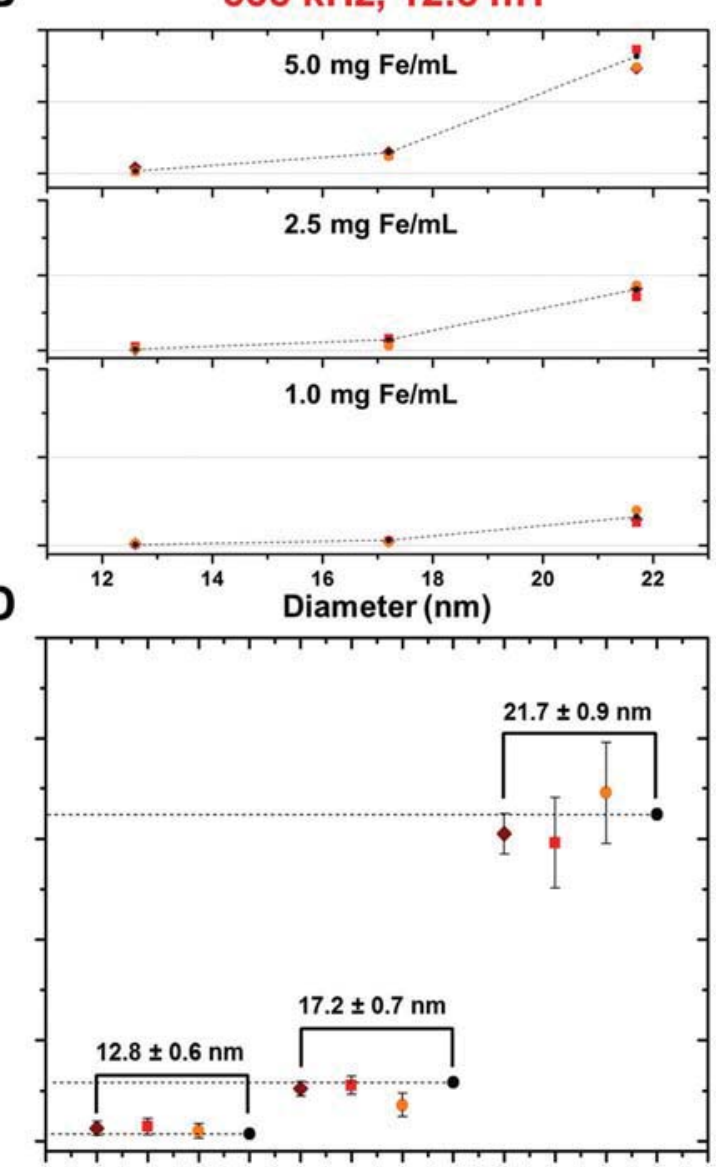

Fig. 4 Slope values extracted from LIT are in agreement with conventional methods and theoretical predictions. SPIONs of three different core sizes at three different iron concentrations were investigated by conventional methods (i.e., fiberoptic cables (FOC) and IR imaging-based standard thermography (ST)) and lock-in thermography (LIT), at two different field strengths and frequencies (A, B). These values were then normalized to concentration and compared to the theoretical values (C, D, mean \pm SD). LIT results in comparatively more precise and reproducible results with lower standard deviations.

Table 1 A summary of the specific absorption rates obtained by different probes. The measured and simulated heating slopes were used to determine the different SAR values and compared to the ones obtained by LIT. These values were consistent with each other, whereas the LIT method exhibited lower standard deviations in almost all cases

\begin{tabular}{|c|c|c|c|c|c|c|c|c|}
\hline & \multicolumn{4}{|l|}{ SAR } & \multicolumn{4}{|l|}{ SAR } \\
\hline & \multicolumn{4}{|c|}{$535 \mathrm{kHz} / 12.5 \mathrm{mT}, \mathrm{W} \mathrm{g}^{-1} \mathrm{Fe}$} & \multicolumn{4}{|c|}{$110 \mathrm{kHz} / 18.0 \mathrm{mT}, \mathrm{W} \mathrm{g}^{-1} \mathrm{~F}$} \\
\hline & LIT & ST & FOC & SIM & LIT & ST & FOC & SIM \\
\hline & \multicolumn{8}{|c|}{$d=12.8 \pm 0.6 \mathrm{~nm}$} \\
\hline Mean & 4.9 & 5.9 & 4.0 & 7.8 & 3.9 & 5.4 & 3.9 & 1.9 \\
\hline \multirow[t]{2}{*}{$\mathrm{SD}$} & 2.6 & 3.4 & 3.0 & 0.0 & 2.8 & 3.5 & 2.2 & 0.0 \\
\hline & \multicolumn{8}{|c|}{$d=17.2 \pm 0.7 \mathrm{~nm}$} \\
\hline Mean & 21.7 & 23.0 & 14.9 & 21.6 & 7.0 & 7.0 & 6.3 & 6.6 \\
\hline \multirow[t]{2}{*}{ SD } & 3.0 & 3.8 & 4.8 & 0.0 & 1.6 & 3.1 & 3.6 & 0.0 \\
\hline & \multicolumn{8}{|c|}{$d=21.7 \pm 0.9 \mathrm{~nm}$} \\
\hline Mean & 127.4 & 123.6 & 144.3 & 128.9 & 62.1 & 58.4 & 56.9 & 61.4 \\
\hline $\mathrm{SD}$ & 8.4 & 18.4 & 21.2 & 0.0 & 3.1 & 9.9 & 15.8 & 0.0 \\
\hline
\end{tabular}




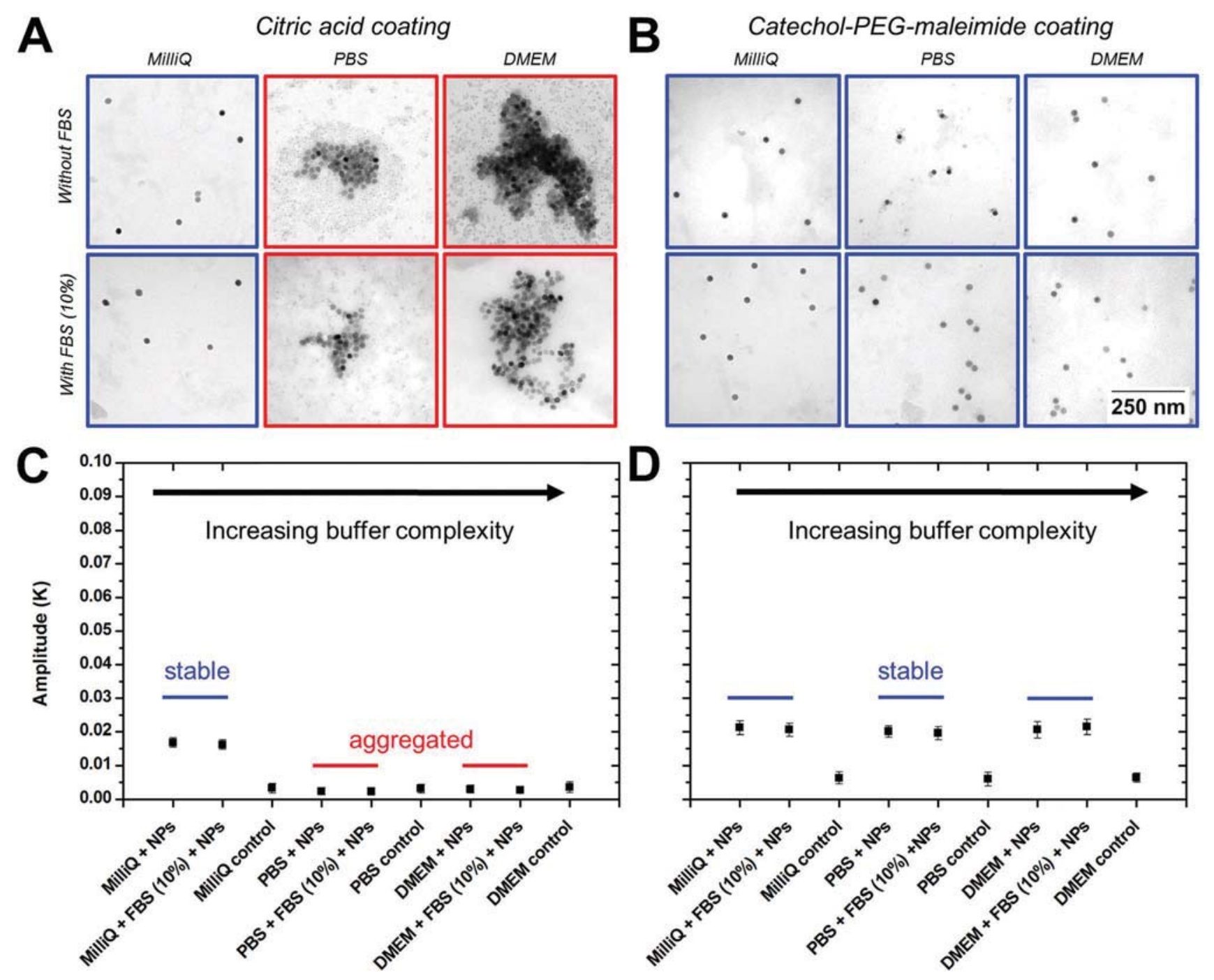

Fig. 5 SPIONs in complex biological environments. Iron oxide NPs coated with either citric acid (A) or catechol-PEG-maleimide (B) were incubated for $24 \mathrm{~h}$ in increasingly complex biological media (with and without the addition of $10 \% \mathrm{FBS}, \mathrm{c}=0.5 \mathrm{mg}$ Fe per $\mathrm{mL}$ ) to study their behaviour in physiological surroundings. They were then investigated by TEM to visualize any differences between aggregated ( $\mathrm{A}$, middle and right columns) and nonaggregated samples (B). The implications of this effect on the heating power of all individual NP batches were then quantified by LIT (C, D), which immediately showed that in this situation, aggregation leads to complete loss of their heating signal (C). In comparison, the NPs, which remained stable, either while still in Milli-Q water (C, left) or in protein-containing surroundings (D), retained their heating power.

Finally, measuring heating performance in liquid suspensions and biological buffers is only a transitional step on the way to therapeutic application, as the properties in these settings are not representative enough to predict their effective behavior in vivo. ${ }^{22,32}$ In this context, SPIONs were embedded in polyacrylamide gels (thickness $=0.5 \mathrm{~mm}$ ), which are regularly employed as tissue phantom models,${ }^{32}$ to move closer to clinically relevant settings. In this manner, the ability of LIT to map the thermal heat distribution and NP behavior in a semisolid state (Fig. 6) was tested, and single-point measurements recorded with fiberoptic cables (Fig. 6A, bottom, 6C) were used as comparisons and controls. Fig. 6C in particular highlights the limitations of these conventional measurements. Besides the fact that measuring within semi-solid samples is cumbersome and invasive (i.e., the probe needs to be inserted into the sample), consistent differences in the heating profiles measured at different points were apparent as well. ${ }^{22}$ Whether these discrepancies occurred over a defined pattern could not be deduced from these data. In contrast, this limitation was circumvented with LIT and the heating behaviour mapped (Fig. 6B) over two dimensions. Once again, the slope values were in accordance with the ones recorded with fiberoptic cables. However, the two-dimensional output offered a more elaborate impression of the sample: the LIT method revealed an inhomogeneous heating pattern over the entire specimen, which was not linked to the NP distribution within it (ESI Fig. $8 \dagger$ ). These investigations were repeated with gels containing different NP concentrations (Fig. 6E), and revealed in this particular case that the non-uniformity in the heating profile was a result of the magnetic field inhomogeneity (Fig. 6F). 

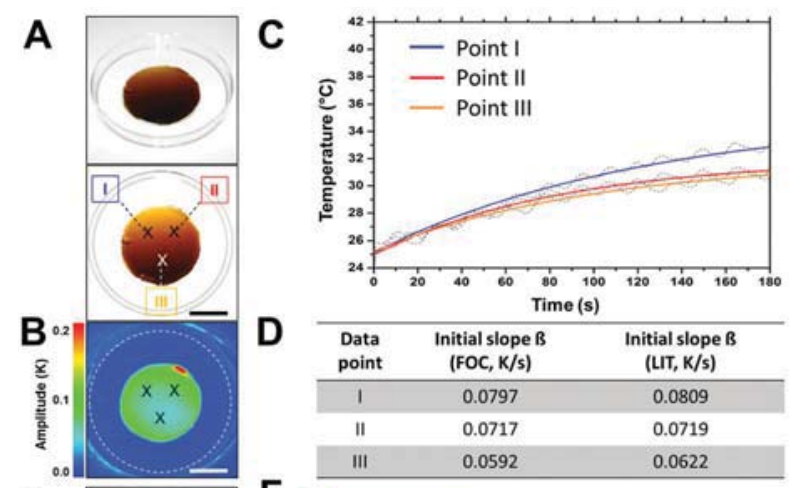

$\mathbf{E}$

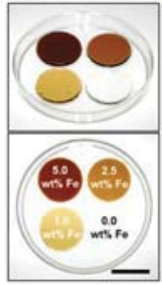

$\mathbf{F}_{0,2}$

\begin{tabular}{ccc}
\hline $\begin{array}{c}\text { Data } \\
\text { point }\end{array}$ & $\begin{array}{c}\text { Initial slope B } \\
\text { (FOC, K/s) }\end{array}$ & $\begin{array}{c}\text { Initial slope B } \\
\text { (LTT, K/s) }\end{array}$ \\
\hline I & 0.0797 & 0.0809 \\
II & 0.0717 & 0.0719 \\
\hline III & 0.0592 & 0.0622 \\
\hline
\end{tabular}

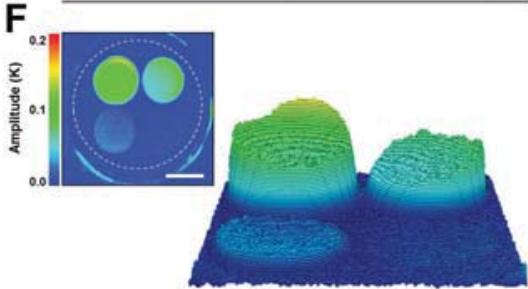

Fig. 6 LIT investigations on SPION-containing substrates highlight the NP heating distribution in a hydrogel as well as heterogeneities in the heating profiles. Investigating the heating power of SPIONs in an embedded state is critical to evaluate their physical behaviour in tissues. In this context, SPION-containing polyacrylamide gels $(A$, scale bar = $10 \mathrm{~mm}$ ) were used as basic models to fix the NPs in a quasi-realistic state. This sample was investigated by LIT (B) and fiberoptic cables (C). The initial heating slopes obtained by both techniques are summarized in (D) (crosses mark the data acquisition points). However, using fiberoptic cables (FOC) underrepresented the overall heating pattern of the sample $(A, C)$, as the values varied considerably when placed in different locations ( $A$, bottom image, crosses signify points of insertion). In contrast, LIT provided a full overview of the sample (B). Again, the heating slopes from the respective points $(B$, crosses signify the extracted pixel values) were directly related to the initial slope values obtained with fiberoptic cables (D). Further investigations of substrates containing varying NP concentrations (E) highlighted inhomogeneities in the heating power related to the AMF (F).

These results stress the difficulty in choosing the proper area of interest while relying on one dimension, as well as perceiving a pattern within the variations. Finally, the complementary simulations (ESI Fig. 7B†) indicated once more that Néel relaxation was the predominant mechanism responsible for heat dissipation from these NPs. Moreover, they emphasized that the LIT method yielded results comparable to standard methods and consistent with simulation results.

\section{Discussion}

The heating efficiency of magnetic NPs is a factor influenced by a myriad of parameters, many of which are not directly linked to the NPs themselves. In this context, iron oxide NPs are paradigms: not all SPIONs are created equal, even when prepared under quasi-identical conditions, ${ }^{13}$ and unavoidable variations, such as in size or crystalline lattice, affect their physical properties. ${ }^{14}$ The agglomeration state, different coat- ings, surrounding environment/buffer, state of dispersion, magnetic field strength, frequency and field heterogeneity are further parameters which dictate the heating performance and hence the SAR. Alas, all these factors render the development of fast, reliable and reproducible characterization protocols pressing to facilitate the optimization of these materials for medical purposes.

In this respect, LIT offers a standardized platform with considerable advantages in addressing these issues. First and foremost, measurements can be performed under quasi-adiabatic conditions, as convective, conductive or radiative phenomena occur on a longer timescale than the modulation period. ${ }^{22}$ Heat loss effects are negligible at high modulation frequencies as well, and bulk heating effects can thus be kept at a minimum. In return, the amplitude signal is not influenced by any of these factors, especially when a sample is homogeneously heated (i.e., at high modulation frequencies). Visualization over a two-dimensional space is critical as well, as it enables the simultaneous analysis of multiple samples, including controls, under identical experimental conditions. With regard to thermal resolution, the signal-to-noise ratio is inversely proportional to the square root of the measurement duration. Accordingly, the sensitivity of the setup can be adjusted by increasing the number of cycles until the desired thermal resolution is reached..$^{28}$ Given the adjustability of the detection limit, very small sample concentrations and volumes are needed, and high spatial resolutions can be reached as well. Although not presented in this study, it has been reported that resolutions of down to $1 \mu \mathrm{m}$ can be reached by using solid immersion lenses. ${ }^{48}$ In the light of these developments, the means to investigating heat signatures of NPs on a cellular level are feasible as well.

With respect to all these benefits, the LIT method is an ideal approach to quantify the heating power, especially the heating slopes, of magnetic NPs. This is particularly apparent when comparing results to standard calorimetric measurements. As demonstrated and repetitively criticised in the literature, ${ }^{15,22}$ standard $\Delta T / \Delta t$ methods are not always the most precise, practical and reproducible approaches. Especially when conducting comparative or analytical studies, uncertainties may be easily introduced during data acquisition and amplified during processing. As shown, many of these concerns are avoided by using LIT. Postprocessing procedures entirely fall away. The measured physical parameter, i.e., the amplitude, is independent, and directly related to the heating slope. Subjective assessments known to affect accuracy, such as on how and where to begin the $\mathrm{fit}^{24}$ are thus bypassed. Consequently, the exact same data can be extracted in a more analytical, statistically relevant and straightforward context while offering a much smaller window to error.

When addressing the NPs, accounting for every variation in the heating power is critical to unfold their maximum potential. With magnetic NPs, the best heating performance is found when the diameter is close to the boundary before undergoing magnetic blocking, where magnetic susceptibility 
is the highest. This was the case with these SPIONs, and the heating slope values measured by LIT were consistent with theoretical predictions. However, it was shown as well how quickly this situation may deteriorate if the materials are compromised, i.e., if they aggregate. While the consequences of this phenomenon on the heating power, although previously addressed, ${ }^{14,42,49-55}$ are still not fully understood, they relate to the basic contributions between relaxation mechanisms. With these SPIONs, Néel relaxation seemed to be the dominating relaxation mechanism. While this assumption is underlined by the results obtained from embedded NPs, the LIT method was pivotal in collecting these data systematically with less uncertainty. In particular with solid samples, standard approaches were troublesome and limited in the information they provided. This was evident in relation to the occurring heterogeneities, which were accounted for by LIT without sacrificing quality or precision. All these results were in agreement with the results of calculations, demonstrating that the LIT method is applicable in the uttermost complex environments, such as gel samples, which are clinically more relevant. $^{32}$

In all, these points not only demonstrate the complexity of these NP systems, but show how their analysis can be facilitated by applying a rapid and standardized method. The direct result is that more precise and extensive information can be obtained on their quality and heating power. ${ }^{32}$ Given how changes in the surroundings affect these parameters - both directly and indirectly - screening them in a systematic way is highly encouraged to ensure maximum therapeutic effectiveness.

\section{Conclusion}

In conclusion, the LIT method yields both quantitative and qualitative information on the thermal effectiveness of iron oxide NPs in any kind of state of matter. Although this study was narrowed down to magnetic NPs, the presented functional principle is applicable to any kind of stimuliresponsive material, provided that the stimulus can be modulated. Given the vast potential of such systems, these results offer an insight into the usefulness and versatility of this method.

\section{Acknowledgements}

This work was supported by the Swiss National Science Foundation (126104, 159803, PP00P2-123373/1 and PP00P2133597/ 1), the Adolphe Merkle Foundation, the University of Fribourg and the Zurich University of Applied Sciences (ZHAW). Parts of the research were supported by the National Centre of Competence in Research Bio-Inspired Materials. The support from the Dr Alfred Bretscher Fund is gratefully acknowledged, and access to TEM was kindly provided by the Microscopy Imaging Centre of the University of Bern.

\section{Notes and references}

1 S. Laurent, D. Forge, M. Port, A. Roch, C. Robic, L. Vander Elst and R. N. Muller, Chem. Rev., 2008, 108, 2064-2110.

2 S. Laurent, S. Dutz, U. O. Häfeli and M. Mahmoudi, Adv. Colloid Interface Sci., 2011, 166, 8-23.

3 R. E. Rosensweig, J. Magn. Magn. Mater., 2002, 252, 370-374.

4 Q. A. Pankhurst, J. Connolly, S. Jones and J. Dobson, J. Phys. D: Appl. Phys., 2003, 36, R167.

5 D. L. Thorek, A. K. Chen, J. Czupryna and A. Tsourkas, Ann. Biomed. Eng., 2006, 34, 23-38.

6 J. Qin, S. Laurent, Y. S. Jo, A. Roch, M. Mikhaylova, Z. M. Bhujwalla, R. N. Muller and M. Muhammed, Adv. Mater., 2007, 19, 1874-1878.

7 A. Jordan, P. Wust, H. Fähling, W. John, A. Hinz and R. Felix, Int. J. Hyperthermia, 2009, 25, 499-511.

8 B. Thiesen and A. Jordan, Int. J. Hyperthermia, 2008, 24, 467-474.

9 C. Bonnaud, C. A. Monnier, D. Demurtas, C. Jud, D. Vanhecke, X. Montet, R. Hovius, M. Lattuada, B. RothenRutishauser and A. Petri-Fink, ACS Nano, 2014, 8, 34513460.

10 M. Mahmoudi, S. Sant, B. Wang, S. Laurent and T. Sen, Adv. Drug Delivery Rev., 2011, 63, 24-46.

11 B. Chertok, B. A. Moffat, A. E. David, F. Yu, C. Bergemann, B. D. Ross and V. C. Yang, Biomaterials, 2008, 29, 487-496.

12 A. Jordan, R. Scholz, P. Wust, H. Fähling and R. Felix, J. Magn. Magn. Mater., 1999, 201, 413-419.

13 M. Kallumadil, M. Tada, T. Nakagawa, M. Abe, P. Southern and Q. A. Pankhurst, J. Magn. Magn. Mater., 2009, 321, 1509-1513.

14 A. E. Deatsch and B. A. Evans, J. Magn. Magn. Mater., 2014, 354, 163-172.

15 I. Andreu and E. Natividad, Int. J. Hyperthermia, 2013, 29, 739-751.

16 R. Hergt, S. Dutz, R. Müller and M. Zeisberger, J. Phys.: Condens. Matter, 2006, 18, S2919.

17 C. S. Kumar and F. Mohammad, Adv. Drug Delivery Rev., 2011, 63, 789-808.

18 B. Mehdaoui, A. Meffre, J. Carrey, S. Lachaize, L. M. Lacroix, M. Gougeon, B. Chaudret and M. Respaud, Adv. Funct. Mater., 2011, 21, 4573-4581.

19 D. L. Leslie-Pelecky and R. D. Rieke, Chem. Mater., 1996, 8, 1770-1783.

20 W. J. Atkinson, I. A. Brezovich and D. P. Chakraborty, IEEE Trans. Biomed. Eng., 1984, 70-75.

21 A. K. Gupta and M. Gupta, Biomaterials, 2005, 26, 39954021.

22 R. Wildeboer, P. Southern and Q. Pankhurst, J. Phys. D: Appl. Phys., 2014, 47, 495003.

23 E. Natividad, M. Castro and A. Mediano, Appl. Phys. Lett., 2008, 92, 093116.

24 S.-Y. Wang, S. Huang and D. Borca-Tasciuc, IEEE Trans. Magn., 2013, 49, 255-262.

25 S. Huang, S. Wang, A. Gupta, D. Borca-Tasciuc and S. Salon, Meas. Sci. Technol., 2012, 23, 035701. 
26 A. Levy, A. Dayan, M. Ben-David and I. Gannot, Nanomedicine, 2010, 6, 786-796.

27 M. Vollmer and K.-P. Möllmann, Infrared thermal imaging: fundamentals, research and applications, John Wiley \& Sons, 2010.

28 O. Breitenstein, W. Warta and M. Langenkamp, Lock-in thermography: Basics and use for evaluating electronic devices and materials, Springer, 2010.

29 P. K. Kuo, T. Ahmed, L. D. Favro, H. Jin and R. L. Thomas, J. Nondestruct. Eval., 1989, 8, 97-106.

30 X. P. V. Maldague, Theory and practice of infrared technology for nondestructive testing, Wiley, 2001.

31 J. Y. Kim, K. S. Chang, M. H. Kook, S. Y. Ryu, H. Y. Choi, K. S. Hong, W. J. Choi, G. Kim, T. H. Jeon and J. Y. Lee, Infrared Phys. Technol., 2013, 57, 76-80.

32 S. Dutz and R. Hergt, Nanotechnology, 2014, 25, 452001.

33 J. Park, K. An, Y. Hwang, J.-G. Park, H.-J. Noh, J.-Y. Kim, J.-H. Park, N.-M. Hwang and T. Hyeon, Nat. Mater., 2004, 3, 891-895.

34 S. Sun and H. Zeng, J. Am. Chem. Soc., 2002, 124, 82048205.

35 M. Lattuada and T. A. Hatton, Langmuir, 2007, 23, 21582168.

36 E. Amstad, T. Gillich, I. Bilecka, M. Textor and E. Reimhult, Nano Lett., 2009, 9, 4042-4048.

37 J. L. Dalsin, L. Lin, S. Tosatti, J. Vörös, M. Textor and P. B. Messersmith, Langmuir, 2005, 21, 640-646.

38 M. Winklhofer and G. T. Zimanyi, J. Appl. Phys., 2006, 99, $08 \mathrm{E} 710$.

39 J. Liu, W. Yang and J. Dai, Infrared Phys. Technol., 2010, 53, 348-357.

40 R. Gupta and O. Breitenstein, Temperature drift correction for fast lock-in infrared thermography, Proceedings 21st European Photovoltaic Solar Energy Conference, ed. J. Poortmans, H. Ossenbrink, E. Dunlop and P. Helm, WIP, Munich, Germany, 2006, pp. 332-335.

41 D. Burnand, C. A. Monnier, A. Redjem, M. Schaefer, B. Rothen-Rutishauser, A. Kilbinger and A. Petri-Fink, J. Magn. Magn. Mater., 2015, 380, 157-162.
42 G. Salas, J. Camarero, D. Cabrera, H. Takacs, M. Varela, R. Ludwig, H. Dahring, I. Hilger, R. Miranda, M. D. Morales and F. J. Teran, J. Phys. Chem. C, 2014, 118, 19985-19994.

43 A. E. Nel, L. Mädler, D. Velegol, T. Xia, E. M. Hoek, P. Somasundaran, F. Klaessig, V. Castranova and M. Thompson, Nat. Mater., 2009, 8, 543-557.

44 T. L. Moore, L. Rodriguez-Lorenzo, V. Hirsch, S. Balog, D. Urban, C. Jud, B. Rothen-Rutishauser, M. Lattuada and A. Petri-Fink, Chem. Soc. Rev., 2015, 44, 6287-6305.

45 K. Rausch, A. Reuter, K. Fischer and M. Schmidt, Biomacromolecules, 2010, 11, 2836-2839.

46 Y. Piñeiro-Redondo, M. Bañobre-López, I. Pardiñas-Blanco, G. Goya, M. A. López-Quintela and J. Rivas, Nanoscale Res. Lett., 2011, 6, 1-7.

47 S. Balog, L. Rodriguez-Lorenzo, C. A. Monnier, M. ObiolsRabasa, B. Rothen-Rutishauser, P. Schurtenberger and A. Petri-Fink, Nanoscale, 2015, 7, 5991-5997.

48 O. Breitenstein, F. Altmann, T. Riediger, D. Karg and V. Gottschalk, Microelectron. Reliab., 2006, 46, 15081513.

49 A. S. Eggeman, S. A. Majetich, D. Farrell and Q. A. Pankhurst, IEEE Trans. Magn., 2007, 43, 2451-2453.

50 C. L. Dennis, A. J. Jackson, J. A. Borchers, R. Ivkov, A. R. Foreman, J. W. Lau, E. Goernitz and C. Gruettner, J. Appl. Phys., 2008, 103.

51 K. D. Bakoglidis, K. Simeonidis, D. Sakellari, G. Stefanou and M. Angelakeris, IEEE Trans. Magn., 2012, 48, 13201323.

52 P. De la Presa, Y. Luengo, M. Multigner, R. Costo, M. P. Morales, G. Rivero and A. Hernando, J. Phys. Chem. C, 2012, 116, 25602-25610.

53 F. J. Teran, C. Casado, N. Mikuszeit, G. Salas, A. Bollero, M. P. Morales, J. Camarero and R. Miranda, Appl. Phys. Lett., 2012, 101.

54 G. Salas, S. Veintemillas-Verdaguer and M. D. Morales, Int. J. Hyperthermia, 2013, 29, 768-776.

55 D. Cabrera, J. Camarero, D. Ortega and F. J. Teran, J. Nanopart. Res., 2015, 17. 